\title{
Erratum to: Scaphoid Nonunion Advanced Collapse: Capitolunate Arthrodesis
}

Matthew Wilson Chapter 24: Scaphoid Nonunion Advanced

Collapse: Capitolunate Arthrodesis, which appears in Scaphoid Fractures and Nonunions, J J. Yao (ed.), Scaphoid Fractures and Nonunions, DOI 10.1007/978-3-319-18977-2_24, contained an error in the author name in both printed and electronic versions. The error has been corrected and is reflected in both print and electronic versions.

The online version of the original chapter can be found under: http://dx.doi.org/10.1007/10.1007/978-3-319-18977-2_24

Matthew Wilson

Carolinas Medical Center, Dept Orthopedic Surgery, Charlotte, NC, USA 